\title{
Management of Takayasu arteritis diagnosed during pregnancy: A case report
}

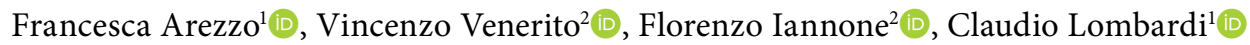 \\ ${ }^{1}$ Department of Obstetrics and Gynecology, University of Bari Aldo Moro, Bari, Italy \\ ${ }^{2}$ Department of Emergency and Organ Transplantations, Rheumatology Unit, University of Bari Aldo Moro, Bari, Italy
}

Takayasu arteritis (TAK) is a rare systemic vasculitis affecting large vessels. Disease onset is insidious, with nonspecific early symptoms contributing to a significant diagnostic and therapeutic delay. TAK usually starts in females of reproductive age; hence, planning and management of pregnancy are important issues. Comprehensive evaluation and a multidisciplinary approach to these patients in a tertiary care center can lead to a favorable outcome for the mother and fetus. Unfortunately, still up to one-third of TAK patients may be advised against pregnancy by their clinicians. ${ }^{1}$ Here, we report the case of a 32-year-old female patient (gravida 2, para 1, $52 \mathrm{~kg}$, body mass index $18.6 \mathrm{~kg} / \mathrm{m}^{2}$ ) with a history of aortic arch and ascending aorta aneurysm. In 2015, the patient had a term vaginal delivery of a fetus with intrauterine growth restriction (birth weight 1900 g). Postpartum period was complicated by the onset of posterior reversible encephalopathy syndrome, six h after delivery. Later, neurological follow-up was negative. She reported to be in good health afterward. In April 2019, she was admitted to Obstetrics and Gynecology departments at 16 weeks of the gestation, complaining of persistent fever unresponsive to paracetamol, paresthesia, and claudication of the left arm and arterial hypertension. Blood tests showed increased acute phase reactants and normal liver and kidney function. A magnetic resonance angiography was carried out, evidencing absence of flow signal in the prevertebral segment of the left subclavian artery with revascularization through the vertebral artery (Figure 1). She was referred to a rheumatologist and TAK was diagnosed (American College of Rheumatology classification criteria were fulfilled because of typical disease onset at $\leq 40$ years, presence of abdominal bruit, left arm claudication, and specific arterial abnormalities). ${ }^{2}$ The therapeutic approach consisted of azathioprine $50 \mathrm{mg}$ twice daily and daily prednisone $50 \mathrm{mg}$ with $6.25 \mathrm{mg}$ reduction per week until reaching a maintenance dose of $6.25 \mathrm{mg}$ daily while alphamethyldopa $500 \mathrm{mg}$ twice daily was administered to control hypertension. The subsequent course of pregnancy proceeded without further complications. Fever resolved three days after glucocorticoids initiation whereas acute phase reactants decreased to normal range. The patient underwent elective caesarean section at 37 weeks of gestation. The postoperative course was regular. Arterial hypertension resolved after the birth. She continued to receive azathioprine at the same dose as it was suitable with breastfeeding. 


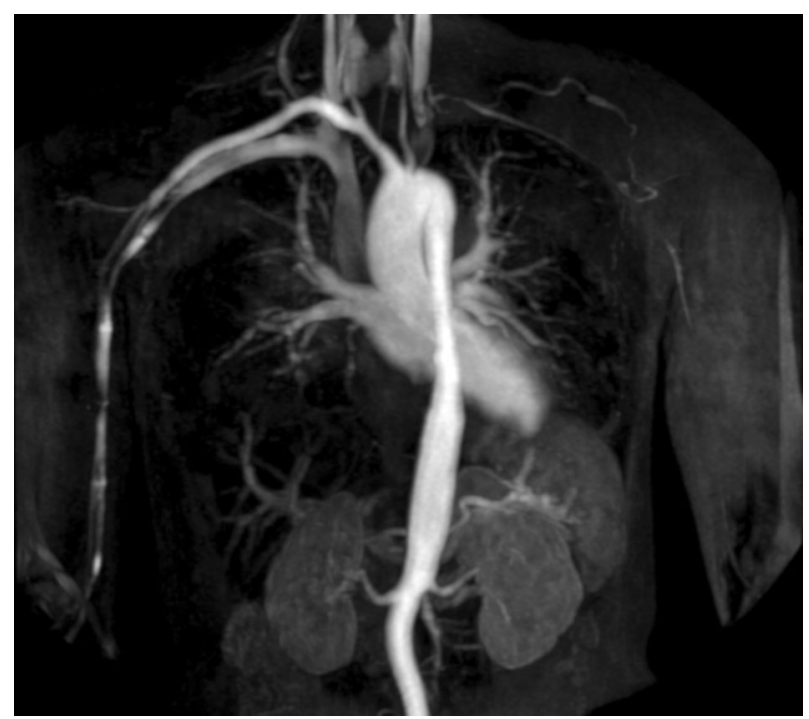

Figure 1. Magnetic resonance angiography showing complete occlusion of prevertebral segment of left subclavian artery. Aneurysms of aortic arch and ascending aorta reported by patient in her anamnesis are also noticeable.

At three-month follow-up, no disease flare occurred. Successful pregnancy outcomes for both mother and fetus are known for TAK patients. ${ }^{3}$ Current evidence indicates that pregnancy does not influence TAK disease activity, although clinicians should be familiar that severe pregnancyrelated complications, such as hypertension, preeclampsia/eclampsia, prematurity, intrauterine growth restriction as well as inflammatory flares have been reported. ${ }^{1,4}$ Of note, their occurrence varies widely between populations; ${ }^{1}$ consequently, uncertainties and fear on the part of both patients and counseling physicians may have an impact on family planning in TAK. ${ }^{1}$ This case offers food for thought capable of informing TAK management in pregnancy. Concerning counseling, patients should be advised to plan pregnancy during states of low disease activity.
Treatment strategies available during pregnancy may include steroids and azathioprine, although there is anecdotal evidence of biologic agents, such as infliximab and tocilizumab, administered until late pregnancy. ${ }^{1,5}$ Clinicians should be aware that blood pressure control is crucial throughout pregnancy. In order to provide a close monitoring for mother and fetus, a multidisciplinary approach involving both rheumatologists and gynecologists is always required. A written informed consent was obtained from the patient.

\section{Declaration of conflicting interests}

The authors declared no conflicts of interest with respect to the authorship and/or publication of this article.

\section{Funding}

The authors received no financial support for the research and/or authorship of this article.

\section{REFERENCES}

1. Gudbrandsson B, Wallenius M, Garen T, Henriksen T, Molberg $\varnothing$, Palm $\varnothing$. Takayasu Arteritis and Pregnancy: A Population-Based Study on Outcomes and Mother/Child-Related Concerns. Arthritis Care Res 2017;69:1384-90.

2. Arend WP, Michel BA, Bloch DA, Hunder GG, Calabrese LH, Edworthy SM, et al. The American College of Rheumatology 1990 criteria for the classification of Takayasu arteritis. Arthritis Rheum 1990;33:1129-34.

3. Alpay-Kanitez N, Omma A, Erer B, Artim-Esen B, Gül A, Inanç M, et al. Favourable pregnancy outcome in Takayasu arteritis: a single-centre experience. Clin Exp Rheumatol 2015;33:S-7-10.

4. Dey M, Kapur A, Goyal S, Wadhwa RD, Srivastava A, Agarwal R. Takayasu arteritis in pregnancy. Med J Armed Forces India 2015;71:S227-9.

5. Dalkilic E, Coskun BN, Yağız B, Pehlivan Y. A successful pregnancy in a patient with Takayasu's arteritis under tocilizumab treatment: A longitudinal case study. Int J Rheum Dis 2019;22:1941-4. 